\title{
Properties of Spontaneous rpsL Mutant of Streptomyces albus KO-1297
}

\author{
A. L. Shemediuk ${ }^{a}$, B. S. Dolia ${ }^{a}$, K. Ochi ${ }^{b}$, V. O. Fedorenko ${ }^{a}$, and B. O. Ostash ${ }^{a}$ * \\ ${ }^{a}$ Ivan Franko National University of Lviv, Lviv, 79005 Ukraine \\ ${ }^{b}$ Hiroshima Institute of Technology, Hiroshima, 731-5193 Japan \\ *e-mail: bohdan.ostash@lnu.edu.ua
}

Received April 29, 2021; revised May 28, 2021; accepted November 18, 2021

\begin{abstract}
The Streptomyces albus J1074 strain remains one of the most popular platforms for the discovery of new natural compounds due to the expression of biosynthetic gene clusters (BGCs) from the microorganisms of the Actinobacteria class. Different methods were tested to provide a maximal expression of heterologous BGCs in this strain. However, there is still no description of the properties of spontaneous J1074 mutants in the $r p s L$ gene encoding a ribosomal protein S12. The interest in such mutations in actinobacteria is due to the fact that they provide a considerable increase in the antibiotic activity. In this work, we describe the isolation and characterization of the $S$. albus KO-1297 strain, which contains a spontaneous missense mutation in the $r p s L$ gene leading to a Lys88Glu substitution in the protein S12. As compared with the initial strain, this mutant exhibits an increased resistance to streptomycin and higher antibiotic productivity. The KO-1297 strain and genetically engineered $r p s L^{\mathrm{K} 88 \mathrm{E}}$ mutant $\mathrm{K} 88 \mathrm{E}$ are not identical in their ability to produce antibiotics. KO-1297 also exhibits a certain level of instability of $r p s L$ mutation. The genomes of KO-1297 and its $r p s L$ WT revertant contain the mutations that can cause phenotypic differences between these strains (as well as between them and SAM2 and K88E strains).
\end{abstract}

Keywords: Streptomyces albus J1074, rpsL mutations, genomics

DOI: $10.3103 / \mathrm{S} 009545272201011 \mathrm{X}$

\section{INTRODUCTION}

The continuous spread of bacterial and viral infections has acquired extraordinary acuteness in the modern world, especially in the light of the pandemic caused by SARS-CoV2 (Editorial 2020), once again reminding mankind about the need to search for new efficient antibacterial and antiviral drugs. The almost inexhaustible potential of gram-positive bacteria of the Actinobacteria class for the production of bioactive substances can be used for the discovery of new antibiotics. Among actinobacteria, the genus Streptomyces is the most studied and commercially successful genus (Pye et al., 2017). To date, the expression of individual genes and genomic libraries of streptomycetes in special platform strains (among which the Streptomyces albus $\mathrm{J} 1074$ strain is the most popular) is methodologically the most efficient approach to the discovery of new bioactive substances. A rapid, dispersed growth and the simplicity of genetic manipulation contributed to the fact that the $S$. albus $\mathrm{J} 1074$ became the "first choice" strain for the expression of heterologous clusters of genes of secondary metabolite biosynthesis in actinobacteria (Bilyk and Luzhetskyy, 2017).

The improvement of biotechnological potential of S. albus $\mathrm{J} 1074$ was an important direction of studies, in the course of which new derivatives of this strain, plasmids for the expression of global regulators of the secondary metabolism were constructed, and different cultivation techniques providing a maximal productivity of this platform were worked out (Lopatniuk et al., 2017; Gummerlich et al., 2021; Kuhl et al., 2021). The selection of highly productive variants among streptomycin-resistant $\left(\mathrm{Str}^{\mathrm{R}}\right)$ Streptomyces mutants is one of the historically first approaches to obtaining overproducers of antibiotics; most frequently, such mutations occur in the rps $L$ gene encoding the ribosomal protein S12 (Shima et al., 1996). Previously, we described a genomic engineering approach to the construction of point rpsL mutations in $\mathrm{J} 1074$, which allowed for the identification of several promising strains (Lopatniuk et al., 2019). This for the first time provided a unique opportunity to objectively compare the peculiarities and advantages of two approaches (a search for spontaneous rpsL mutants and the construction of such mutants using genomic engineering methods. The important question is whether only the rps $L$ mutation (without additional mutations) causes an increase in antibiotic productivity in spontaneous mutants. Moreover, although some consequences of rps $L$ mutations are known (for example, a higher level of protein synthesis in a stationary phase) (Okamoto-Hosoya 
et al., 2003), a complete mechanism of the influence of these mutations on the secondary metabolism of Streptomyces remains unclear. Genomic engineering theoretically allows one to obtain certain changes in the genome in a "pure" form, that is, without accompanying mutations. The comparison of isogenic rps $L$ mutants of different origin is one of the ways to find answers to the above questions. Correspondingly, we describe the isolation and initial characterization of the spontaneous $S$. albus KO-1297 rpsL mutant in this work. The obtained results indicate that biotechnologically valuable properties (for example, the ability to produce antibiotics) of genetically engineered and spontaneous mutants, isogenic only for the $r p s L$ allele, can differ. This is probably caused by the presence of additional mutants in the genomes of spontaneous mutants. We provide the results from analyzing the KO-1297 strain genome that serve as evidence of the above statement.

\section{MATERIALS AND METHODS}

Streptomyces albus SAM2 (also known as $\Delta \mathrm{pse}$ ), S. albus J1074 derivative with a deletion of pseudo$a_{t t B}{ }^{\varphi C 31}$ site (Bilyk and Luzhetskyy, 2014), was used in all experiments as the original strain. The $S$. albus $\mathrm{K} 88 \mathrm{E}$ mutant contains the $r p s L^{\mathrm{K} 88 \mathrm{E}}$ allele obtained by the methods of genomic engineering (Lopatniuk et al., 2019). The KO-1297 strain was selected among the spontaneous SAM2 $\mathrm{Str}^{\mathrm{R}}$ mutants as described below. In this work, the KO-1297L strain was obtained as a spontaneous streptomycin-sensitive $\left(\mathrm{Str}^{\mathrm{S}}\right)$ KO-1297 derivative. Streptomycetes were cultivated on TSA, GYM, SG2, TSB, and SFM media (Tanaka et al., 2009; Koshla et al., 2017; Koshla et al., 2019) at $30^{\circ} \mathrm{C}$. The strains Bacillus cereus ATCC19637, Staphylococcus aureus 209P, and Debaryomyces hansenii VKM9 were used as test culture for determining the antibiotic activity of rps $L$ mutants. The cosmid pOJara with aranciamycin biosynthesis gene cluster was described by Koshla et al. (2017). The agar block method for determining antibiotic activity of $S$. albus strains, extraction, and spectrophotometry of aranciamycins were also described in Koshla et al. (2017). All components of culture media were purchased in Conda Laboratories. Streptomycin sulphate was purchased in Roth. Other reagents from the ChemLaborReactive Company were of the level of purity not less than chemically pure.

The resistance of $S$. albus clones to streptomycin was determined by the method of colony replicas on agar SFM medium with antibiotic. To determine a minimum inhibitory concentration (MIC) of streptomycin, spore suspensions of the studied strains $\left(10^{5} \mathrm{cfu}\right)$ were seeded in the wells of an immunological plate from $120 \mu \mathrm{L}$ of TSB medium containing different concentrations of antibiotic (from 0.6 to $200 \mu \mathrm{m}$ ). The plates were incubated on a shaker and growth was determined after $48 \mathrm{~h}$ of cultivation.

Primers used for screening and identification of mutations in the rps $L$ gene and the conditions for PCR screening of such mutations were described in (Shima et al., 1996). Genomic DNA of KO-1297 and KO$1297 \mathrm{~L}$ strains was isolated by a salting method according to the procedure no. 4 described in (Kieser et al., 2000) from 24-h cultures grown in tryptic soy broth (TSB) medium. The concentration and quality of isolated DNA were determined on a Trinean Xpose microchannel analyzer (Gentbrugge, Belgium). The quality control DNA was used to construct a library for sequencing by the Illumina method (TruSeq DNA PCR-Free Kit). The library was sequenced on an Illumina HiSeq instrument.

The initial data of sequenced $S$. albus genomes, reference sequence of the $\mathbf{J} 1074$ genome are located in a database maintained by the group of Lviv University: https://biotools.online/media/. At the stage of data quality control, all original genome reads were tested for the quality level and for the presence of sequencing adapters using the FastQC program (http://www.bioinformatics.babraham.ac.uk/projects/fastqc). Poor quality sequences were extracted using the Trimmomatic v. 0.36 program. The genome reads were aligned against the reference sequence of J1074 genome (accession number CP004370) using a Bowtie2 v. 2.2.5. Polymorphisms (SNP) and indels (DIP) were detected using a ReadXplorer (Hilker et al., 2014). The coverage level of sequenced genome was 55:165-fold level (see the list on the above mentioned database webpage: Supplementary_data_(avg_coverage).xlsx?, coverage level and data quality see also xlsx files in the SNP folder of the above mentioned online folder).

\section{RESULTS}

Isolation KO-1297 and KO-1297L strains. In this study, we focused on generation a spontaneous $S$. albus mutant containing a point substitution in the rps $L$ gene, which leads at the level of the amino acid sequence of the protein $\mathrm{S} 12$ to the substitution of a lysine residue at position 88 by a glutamate residue $\left(r p s L^{\mathrm{K} 88 \mathrm{E}}\right.$ allele). This is one of the most studied mutant alleles of the rps $L$ gene, which causes a significant increase in the production of antibiotics in streptomycetes (Shima et al., 1996; Okamoto-Hosoya et al., 2003; Ochi, 2017). A search for such a mutant was performed by inoculation of spore suspensions (approximately $10^{8}$ colony-forming units, cfu) of the initial SAM2 strain on the agar medium GYM containing $100 \mu \mathrm{g} / \mathrm{mL}$ streptomycin and PCR screening of colonies that grew on these plates for 7 days. In total, we identified seven $\mathrm{Str}^{\mathrm{R}}$ clones that did not lost their resistance up to $100 \mu \mathrm{g} / \mathrm{mL}$ streptomycin after three passages under nonselective conditions. The frequency of their occurrence was $1.4 \times 10^{-8}$. One of the 
Table 1. Antibiotic activity ${ }^{1}$ of $S$. albus strains

\begin{tabular}{l|c|c|c}
\hline \multicolumn{1}{c|}{ S. albus strains } & B. cereus & Staph. aureus & D. hansenii \\
\hline SAM2 & +2 & + & + \\
K88E & ++ & ++ & + \\
KO-1297 & + & ++ & ++ \\
KO-1297L & + & + & + \\
\hline
\end{tabular}

clones designated as $\mathrm{KO}-1297$ contained the substitution of adenine residue with guanine at position 262 of the rpsL gene ( $x n r_{-} 3720$ ), which leads to the desired substitution $\mathrm{K} 88 \mathrm{aE}$ at the level of amino acid sequence.

We decided to study in more detail the stability of streptomycin resistance phenotype of KO-1297. For this purpose, we performed 13 passages of the culture of this mutant in a liquid TSB medium in the absence of selective pressure, and the suspension of mycelial cells after the last passage was seeded in dilutions on mannitol soya flour (SFM) agar. Among approximately 10000 colonies grown on SFM, a single colony not growing on SFM with $100 \mu \mathrm{g} / \mathrm{mL}$ streptomycin was found by the replica method. The obtained clone was designated as KO-1297L. We determined minimum inhibitory concentrations (MIC) of streptomycin for the strains SAM2, K88E, KO-1297, and KO-1297L. The following MIC were established: $1.6 \mu \mathrm{g} / \mathrm{mL}$ for SAM and $6.25 \mu \mathrm{g} / \mathrm{mL}$ for $\mathrm{KO}-1297 \mathrm{~L}$. A growth of K88E and KO-1297 was observed by the highest studied streptomycin concentration $(200 \mu \mathrm{g} / \mathrm{mL})$. Consequently, although KO-1297L lost the ability to grow due to high concentrations of streptomycin, it differs from SAM2 in streptomycin resistance phenotype and, obviously, is not a revertant to the wild type by this trait.

Antibiotic activity of rps $L$ mutants. We grew the studied mutants on GYM and SG2 media and used 5day agar blocks of these cultures to estimate the antibiotic activity of these strains against a number of test cultures. The results of these experiments are summarized in Table 1 and Fig. 1a. It is noteworthy that mutants (both spontaneous and genetically engineered K88E) mostly demonstrate the highest antibiotic activity against used bacterial cultures and $D$. hansenii yeasts, while the revertant rather resembles the original SAM2 strain. However, there are also differences between the spontaneous and genetically engineered mutants that carry the $r p s L^{\mathrm{K} 88 \mathrm{E}}$ allele. Thus, the former is less active against B. cereus but is more active with regard to $D$. hansenii. Differences between the studied $r p s L^{\mathrm{K} 88 \mathrm{E}}$ mutants are also traced with heterologous expression of aranciamycin biosynthesis gene cluster in these strains. Thus, the spontaneous $r p s L^{\mathrm{K} 88 \mathrm{E}}$ mutant KO-1297 demonstrated much higher ability to produce the above-mentioned antibiotic as compared with genetically engineered K88E strain on agar SFM medium (Fig. 1b) and in liquid SG2 medium. As for the last case, KO-1297 produced almost one and a half and three times more aranciamycin $(0.485 \mathrm{cu}$, data on spectrophotometry of extracts) as compared with the K88E strain $(0.339 \mathrm{cu})$ and SAM $(0.159 \mathrm{cu})$, respectively.

Analysis of genomes of KO-1297 and KO-1297L. The above-described results induced us to sequence the genomes of parental SAM2 strain and KO-1297 and $\mathrm{KO}-1297 \mathrm{~L}$ mutants in order to understand the genetic basis of differences in antibiotic resistance and antibiotic activity identified in them. As a result of mapping sequencing data in relation to the reference genome J1074, the mutations containing KO-1297 and $\mathrm{KO}-1297 \mathrm{~L}$ as compared with SAM2 were found. The list of differences is given in Table 2. The original data are available in the database at the link provided in the Materials and Methods section. A deletion in the phosphodiesterase gene $\left(x n r_{-} 1338\right)$, which can be involved in metabolism of c-di-ĞMP secondary messenger controlling secondary metabolism and morphogenesis of streptomycetes, became an interesting finding in the KO-1297 genome. The loss of $r p s L^{\mathrm{K} 88 \mathrm{E}}$ mutation in KO-1297L genome was confirmed. At the same time, four new mutations were detected in KO- $1297 \mathrm{~L}$, out of which the mutation in the transcription factor gene $x n r_{-} 4701$ is, in our opinion, the most interesting for the reasons described below in the Discussion section. The protein Xnr_4701 from the IclR family consists of 262 amino acid residues. Using the https://smart.embl.de/ service, it was found that the Lys136aGlu substitution, caused by a mutation in the $x n r_{-} 4701$, is located outside the DNA-binding domain, which covers a protein segment from 2 to 89 amino acid residues and, consequently, should not violate its ability to interact with DNA.

\section{DISCUSSION}

The $S$. albus $\mathrm{J} 1074$ strain is becoming an increasingly important platform for the discovery of biologically active substances (Olano et al., 2014; Kallifidas et al., 2018), which causes a significant interest in studying all aspects of its biology (Ahmed et al., 2017; Myronovskyi et al., 2018; Koshla et al., 2019a). Spontaneous (or mutagen-induced) streptomycin-resistant mutants, arising as a result of point substitutions in the $r p s L$ gene of ribosomal protein $\mathrm{S} 12$, frequently exhibit an increased ability to produce antibiotics. The exact mechanism of the effect of $r p s L$ on the production of 
(a)

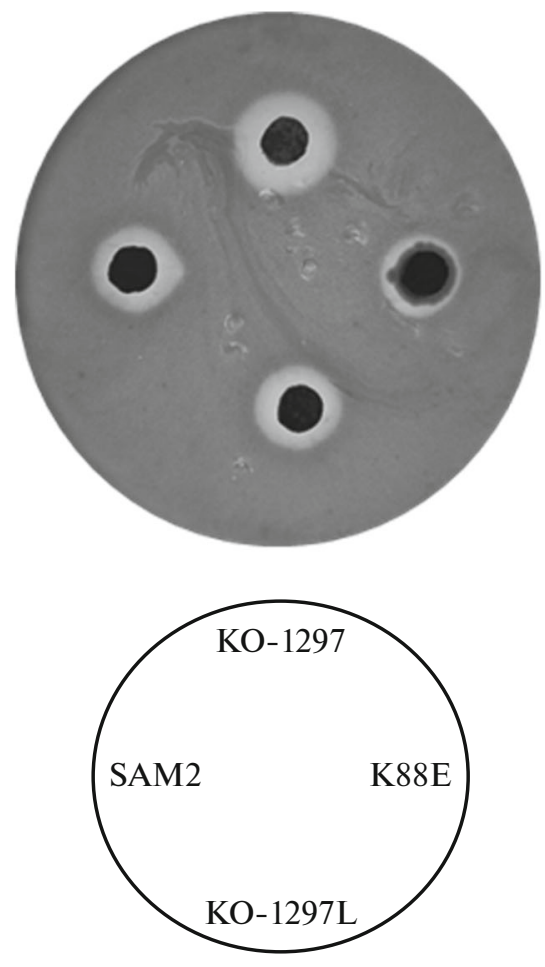

(b)

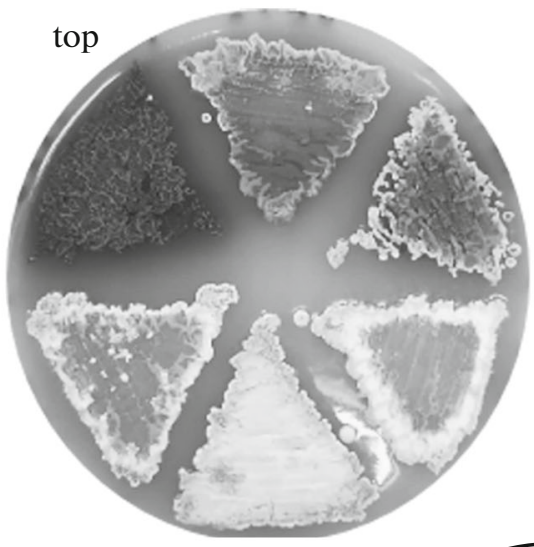

bottom

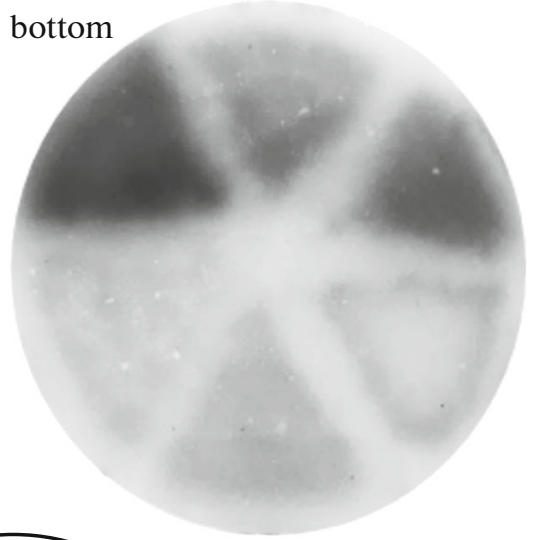

Fig. 1. Antibiotic activity of $S$. albus rps $L$ mutants. (a) Suppression of $D$. hansenii growth by agar blocks of $S$. albus strains; (b) heterologous production of aranciamycin (red color) by $S$. albus strains. For location of strains, see graphic legends under the photos. The photos demonstrate a typical result of at least five repetitions.

secondary metabolites remains unknown (see Introduction). To date, there are no works in which they would try to study the whole genome of the spontaneous rpsL mutant and to detect other mutations that can be carried by such a mutant. The involvement of namely rps $L$ mutations in the overproduction of antibiotics is also indicated by the study of $r p s L$ mutants of $S$. albus that were obtained by genomic engineering methods (Lopatniuk et al., 2019), where the probability of side mutation events is lower. However, the advantage of obtaining and using namely genetically engineered mutants in biotechnology remains unclear.
Theoretically, a positive effect of $r p s L$ mutations on the secondary metabolism of streptomycetes can be maximally manifested with the occurrence of other mutations that can be selected during screening the spontaneous mutants. The results of studying the spontaneous KO-1297 rps $L^{\mathrm{K} 88 \mathrm{E}}$ mutant in this work provide the first evidence in favor of such a scenario. As compared with genetically engineered $\mathrm{K} 88 \mathrm{E} r p s L^{\mathrm{K} 88 \mathrm{E}}$ strain, KO-1297 produces more endogenous antifungal compounds and is able to accumulate more aranciamycin with heterologous expression of the genes of its biosynthesis. It is interesting that KO-1297 contains

Table 2. List of new mutations in KO-1297 and KO-1297L genomes as compared with parental SAM2 genome

\begin{tabular}{|c|c|c|c|c|}
\hline \multirow{2}{*}{ Mutation } & \multirow{2}{*}{ Locus $^{1}$} & \multirow{2}{*}{ Function } & \multicolumn{2}{|c|}{ Presence of mutation in: } \\
\hline & & & KO- 1297 & KO-1297L \\
\hline$\Delta 1595677$ & $x n r_{-} 1338$ & Phosphodiesterase & + & - \\
\hline $\operatorname{Trp} \rightarrow$ Cys & $x n r_{-} 2696$ & Phage integrase & - & + \\
\hline $\mathrm{Ala} \rightarrow \mathrm{Ser}$ & $x n r \_2758$ & Asp dehydrogenase & + & - \\
\hline Gln $\rightarrow$ Arg & $x n r_{-} 3600$ & Hypothetical protein & - & + \\
\hline Lys $\rightarrow$ Glu & $x n r \_3720$ & Ribosomal protein S12 & + & - \\
\hline Lys $\rightarrow$ Glu & $x n r_{-} 4071$ & Transcription factor of IclR family & - & + \\
\hline
\end{tabular}

\footnotetext{
${ }^{1}$ Type and localization of missense mutation in the gene are given in the supplementary materials; see hyperlink in Materials and Methods.
} 
the additional mutation as compared with SAM2 (in the phosphodiesterase gene $x n r_{-} 1338$ ). The involvement of homologues of this gene in the secondary metabolism of streptomycetes is well known (Makitrynskyy et al., 2020). A possible involvement of this gene in the secondary metabolism of $S$. albus and sequencing of the genome of genetically engineered K88E mutant are the subject of our current studies. It is possible that this mutant contains additional mutations and not only those introduced by genomic engineering methods that can affect its phenotype.

A quite simple (in terms of frequency of appearance) detection of KO-1297L revertant was another interesting result of our study. In the scientific literature, there are no data on the frequency of reversion of $r p s L$ mutants of streptomycetes. In our case, this frequency is rather high, which obviously indicates a high biological cost of rps $L$ mutation. It is known that the $r p s L^{\mathrm{K} 88 \mathrm{E}}$ mutation increases the accuracy of protein synthesis and thus slows down the growth of streptomycete cultures (Shima et al., 1996; Okamoto-Hosoya et al., 2003; Tanaka et al., 2009). This can cause a selective advantage of reversion. However, a rapid occurrence of the KO-1297L strain can be namely caused by the KO-1297 genotype and not reflect a general trend for all rpsL mutants. For example, the presence of a mutation in the phage integrase gene in KO-1297 can cause genomic rearrangements promoting reversions. The value of the metabolic burden that $r p s L^{\mathrm{K} 88 \mathrm{E}}$ mutation imposes on the strain is determined by a complex of all mutations in the strain $\left(r p s L^{\mathrm{K} 88 \mathrm{E}}\right.$ and accompanying mutations), a cumulative effect of which is impossible to predict only on the basis of bioinformatic analysis (Westhoff et al., 2017).

Despite the loss of the $r p s L^{\mathrm{K} 88 \mathrm{E}}$ mutation, KO-1297L is more resistant to streptomycin than the parental SAM2 strain. We assume that this is caused by a missense mutation in the transcription factor gene of the IclR family ( $\left.x n r_{-} 4701\right)$. IclR-like proteins were described, particularly, $\mathrm{NdgR}$ regulator (a regulator of growth dependent on nitrogen and antibiotic production), which has a global effect on the synthesis of amino acids, morphogenesis, and production of antibiotics in Streptomyces coelicolor (Yung-Hun et al., 2009). NdgR homologues were found in other Streptomyces species as well as in mycobacteria and corynebacteria, which indicates its role as a global regulator. It is possible that the mutation in $x n r_{-} 4701$ leads to the formation of the protein that functions constitutively or recognizes new gene promoters that way provide resistance to streptomycin in a certain. Verification of this assumption is the subject of our current experiments that can lead to the identification of new mechanisms of streptomycin resistance.

\section{ACKNOWLEDGMENTS}

We are grateful to J. Kalinovski (Bielefeld University, Federal Republic of Germany), T. Gren, and T. Weber (Technical University of Denmark) for providing the opportunity for sequencing of $S$. albus strains.

\section{FUNDING}

This study was supported by the Ministry of Education and Science of Ukraine (grant nos. Bg-09F, Bg-80F, BF-2021).

\section{COMPLIANCE WITH ETHICAL STANDARDS}

The authors declare that they have no conflict of interests. This study does not contain any experiments involving animals, human participants, animal or human cell lines.

\section{REFERENCES}

Ahmed, Y., Rebets, Y., Tokovenko, B., Brotz, E., and Luzhetskyy, A., Identification of butenolide regulatory system controlling secondary metabolism in Streptomyces albus J1074, Sci. Rep., 2017, vol. 7, p. 9784. https://doi.org/10.1038/s41598- 017-10316-y

Bilyk, B. and Luzhetskyy, A., Unusual site-specific DNA integration into the highly active pseudo-attB of the Streptomyces albus J1074 genome, Appl. Microbiol. Biotechnol., 2014, vol. 98, pp. 5095-5104.

https://doi.org/10.1007/ s00253-014-5605-y

Bilyk, O. and Luzhetskyy, A., Host organism: Streptomyces, in Industrial Biotechnology, Wittmann, Ch. and Liao, J.C., Eds., Weinheim, Germany: Wiley-VCH Verlag GmbH \& Co KGaA, 2017, chapter 13, pp. 487-506. https://doi.org/10.1002/9783527807796.ch13

Editorial, Antimicrobial resistance in the age of COVID-19, Nat. Microbiol., 2020, vol. 5, p. 779.

https://doi.org/10.1038/ s41564-020-0739-4

Gummerlich, N., Manderscheid, N., Rebets, Y., Myronovskyi, M., Glaser, L., Kuhl, M., Wittmann, C., and Luzhetskyy, A., Engineering the precursor pool to modulate the production of pamamycins in the heterologous host S. albus J1074, Metab. Eng., 2021, vol. 67, pp. $11-18$. https://doi.org/10.1016/j.ymben.2021.05.004

Hilker, R., Stadermann, K., Doppmeier, D., Kalinowski, J., Stoye, J., and Straube, J., ReadXplorer-visualization and analysis of mapped sequences, Bioinformarics, 2014, vol. 30, pp. 2247-2254.

https://doi.org/10.1093/bioinformatics/btu205

Kallifidas, D., Jiang, G., Ding, Y., and Luesch, H., Rational engineering of Streptomyces albus J1074 for the overexpression of secondary metabolite gene clusters, Microb. Cell Fact., 2018, vol. 17, pp. 25-37. https://doi.org/10.1021/acschembio.7b00359

Kieser, T., Bibb, M.J., Buttner, M.J., Chater, K.F., and Hopwood, D.A., Practical Streptomyces Genetics, Norwich, United Kingdom: John Innes Foundation, 2000. ISBN 0-7084-0623-8.

Koshla, O., Lopatniuk, M., Rokytskyy, I., Yushchuk, O., Dacyuk, Y., Fedorenko, V., Luzhetskyy, A., and Ostash, B., Properties of Streptomyces albus J1074 mutant 
deficient in tRNALeuUAA gene bldA, Arch. Microbiol., 2017, vol. 199, pp. 1175-1183.

https://doi.org/10.1007/s00203- 017-1389-7

Koshla, O., Rokytskyy, I., Ostash, I., Busche, T., Kalinowski, J., Mosker, E., Sußmuth, R., Fedorenko, V., and Ostash, B., Secondary metabolome and transcriptome of Streptomyces albus J1074 in liquid medium SG2, Cyt. Genet., 2019, vol. 53, pp. 1-7. https://doi.org/10.3103/S0095452719010080

Koshla, O., Yushchuk, O., Ostash, I., Dacyuk, Y., Myronovskyi, M., Jäger, G., Süssmuth, R.D., Luzhetskyy, A., Bystrom, A., Kirsebom, L.A., and Ostash, B., Gene miaA for post-transcriptional modification of tRNAXXA is important for morphological and metabolic differentiation in Streptomyces, Mol. Microbiol., 2019a, vol. 112, pp. $249-265$. https://doi.org/10.1111/mmi.14266

Kuhl, M., Ruckert, C., Glaser, L., Beganovic, S., Luzhetskyy, A., Kalinowski, J., and Wittmann, C., Microparticles enhance the formation of seven major classes of natural products in native and metabolically engineered actinobacteria through accelerated morphological development, Biotechnol. Bioeng., 2021. https://doi.org/10.1002/ bit.27818

Lopatniuk, M., Myronovskyi, M., and Luzhetskyy, A., Streptomyces albus: a new cell factory for non-canonical amino acids incorporation into ribosomally synthesized natural products, ACS Chem. Biol., 2017, vol. 12, art. 23622370.

Lopatniuk, M., Myronovskyi, M., Nottebrock, A., Busche, T., Kalinowski, J., Ostash, B., Fedorenko, V., and Luzhetskyy, A., Effect of "ribosome engineering" on the transcription level and production of $S$. albus indigenous secondary metabolites, Appl. Microbiol. Biotechnol., 2019, vol. 103, pp. 7097-7110.

https://doi.org/10.1007/s00253-019- 10005-y

Makitrynskyy, R., Tsypik, O., Nuzzo, D., Paululat, T., Zechel, D.L., and Bechthold, A., Secondary nucleotide messenger c-di-GMP exerts a global control on natural product biosynthesis in streptomycetes, Nucleic Acids Res., 2020, vol. 48, pp. 158-1598. https://doi.org/10.1093/nar/gkz1220

Myronovskyi, M., Rosenkranzer, B., Nadmid, S., Pujic, P., Normand, P., and Luzhetskyy, A., Generation of a cluster-free Streptomyces albus chassis strains for improved heterologous expression of secondary metabolite clusters, Metab. Eng., 2018, vol. 49, pp. 316-324. https://doi.org/10.1016/j.ymben.2018.09.004
Ochi, K., Insights into microbial cryptic gene activation and strain improvement: principle, application and technical aspects, J. Antibiot., 2017, vol. 70, pp. 25-40. https://doi.org/10.1038/ja.2016.82

Olano, C., Garcia, I., Gonzalez, A., Rodriguez, M., Rozas, D., Rubio, J., Sanchez-Hidalgo, M., Braca, A.F., Mendez, C., and Slas, J.A., Activation and identification of five clusters for secondary metabolites in Streptomyces albus J1074, Microbiol. Biotechnol., 2014, vol. 7, pp. 242-256. https://doi.org/10.1111/1751-7915.12116

Okamoto-Hosoya, Y., Hosaka, T., and Ochi, K., An aberrant protein synthesis activity is linked with antibiotic overproduction in rpsL mutants of Streptomyces coelicolor A3(2), Microbiology, 2003, vol. 149, pp. 3299-3309. https://doi.org/10.1099/mic.0.26490-0

Pye, C., Bertin, M., Lokey, R., Gerwick, W., and Linington, R., Retrospective analysis of natural products provides insights for future discovery trends, Proc. Natl. Acad. Sci. U. S. A., 2017, vol. 114, pp. 5601-5606. https://doi.org/10.1073/pnas.1614680114

Shima, J., Hesketh, A., Okamoto, S., Kawamoto, S., and Ochi, K., Induction of actinorhodin production by rpsL (encoding ribosomal protein S12) mutations that confer streptomycin resistance in Streptomyces lividans and Streptomyces coelicolor A3(2), J. Bacteriol., 1996, vol. 178, art. 7276-7284. https://doi.org/10.1128/jb.178.24.7276-7284.1996

Tanaka, Y., Komatsu, M., Okamoto, S., Tokuyama, S., Kaji, A., Ikeda, H., and Ochi, K., Antibiotic overproduction by rpsL and $r \operatorname{sm} G$ mutants of various actinomycetes, Appl. Environ. Microbiol., 2009, vol. 75, pp. 4919-4922. https://doi.org/10.1128/AEM.00681-09

Westhoff, S., van Leeuwe, T.M., Qachach, O., Zhang, Z., van Wezel, G.P., and Rozen, D.E., The evolution of no-cost resistance at sub-MIC concentrations of streptomycin in Streptomyces coelicolor, ISME J., 2017, vol. 11, pp. 1168-1178.

https://doi.org/10.1038/ismej.2016.194

Yung-Hun, Y., Eunjung, S., Eun-Jung, K., Kwangwon, L., Woo-Seong, K., Sung-Soo, P., Ji-Sook, H., and Byung-Gee, K., NdgR, an IclR-like regulator involved in amino-acid-dependent growth, quorum sensing, and antibiotic production in Streptomyces coelicolor, Appl. Microbiol. Biotechnol., 2009, vol. 9, pp. 501-511. https://doi.org/10.1007/s00253-008-1802-X

Translated by A. Barkhash 